Review

\title{
Light-Activated Local Drug Delivery From Hydrogels: Potential for GBM Treatment
}

Lina Nguyen *, Gabrielle Romena, Ethan Nguyen, Cassandra Tran, Kaitlyn Nguyen, Ananya Devarajan, Henry Hirschberg

Beckman Laser Institute and Medical Clinic, University of California, Irvine 1002 Health Sciences Rd, Irvine, CA, 92617, United States; E-Mails: linanguyen824@gmail.com; gromena@uci.edu;

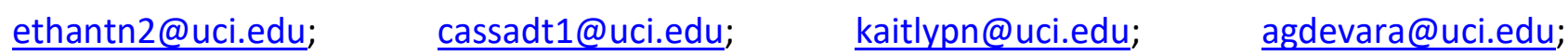
hhirschb@hs.uci.edu

* Correspondence: Lina Nguyen; E-Mail: linanguyen824@gmail.com

Academic Editor: Bart Ellenbroek

Special Issue: Novel Approaches to Glioblastoma

OBM Neurobiology

2021, volume 5 , issue 1

doi:10.21926/obm.neurobiol.2101090
Received: December 15, 2020

Accepted: March 15, 2021

Published: March 23, 2021

\begin{abstract}
Following post-operative treatment, the vast majority of GBMs recur, usually within $2 \mathrm{~cm}$ of the tumor resection margin. Since these tumors seldom metastasize outside the CNS, improved localized therapies should prove beneficial. Drug delivery systems (DDS) that allow control over drug release by an externally applied energy, such as light, have the potential of improving the results of chemotherapy. Light-activated hydrogel DDS, implanted in the operative cavity formed by tumor resection, bypasses the blood brain barrier and allows for a precise spatio-temporal control of drug release or drug activation. Localized, on-demand release of anticancer drugs to normal brain infiltrated by tumor cells should therefore improve therapeutic efficacy and minimize systemic, drug-related side effects. Various forms of light-activated hydrogels have utilized near infrared, visible or ultraviolet wavelength irradiation. One form of hydrogel, fibrin glue (FG), composed of a combination of fibrinogen and thrombin, has several characteristics that make it well-suited for this form of sustained release drug delivery. Unlike most synthetic hydrogels, FG is a natural product and has been
\end{abstract}

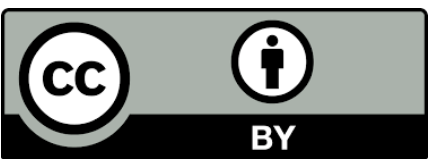

(C) 2021 by the author. This is an open access article distributed under the conditions of the Creative Commons by Attribution License, which permits unrestricted use, distribution, and reproduction in any medium or format, provided the original work is correctly cited. 
used clinically in surgery for decades, so its biocompatibility and lack of toxicity has been clearly proven. The light-driven drug activation technology, photochemical internalization $(\mathrm{PCl})$ has been shown to enhance the intracellular efficacy of chemotherapeutic agents released from fibrin glue. A summary of the light-activated hydrogel techniques developed for the site and temporal-specific delivery of chemotherapy for the treatment of GBM will be discussed in this review.

\section{Keywords}

Hydrogel; light activation; photochemical internalization; fibrin glue; gliomas

\section{Background}

Effective treatment of glioblastoma multiforme (GBM) remains an unreached goal. Surgical tumor resection is usually mandatory in the treatment of gliomas primarily to decompress the brain and obtain a pathological diagnosis. Employing the improved imaging and surgical techniques now available, the incidence of gross tumor resection, as seen on postoperative magnetic resonance imaging (MRI), has greatly increased [1, 2]. Unfortunately, owing to the highly infiltrative nature of GBM that leads to indistinct tumor boundaries, surgical intervention is never curative in adult patients. Standard, postoperative adjuvant therapies, consisting of radiation and chemotherapy, most often fail to eliminate infiltrating glioma cells in or beyond the brainadjacent-to-tumor (BAT) region - a zone that commonly extends several centimeters from the resection margin. This is thought to be the reason for the extremely high rate of tumor recurrence, approaching $100 \%$. Since $80 \%-90 \%$ of GBMs recur within $2 \mathrm{~cm}$ of the resection margin $[3,4]$ and seldom metastasize outside the CNS, a reasonable starting point for improving the prognosis of GBM patients would be the development of improved local therapies. These should be capable of eradicating remaining glioma cells in the tumor resection margin and infiltrating cells in the brainadjacent-to-tumor BAT region.

In this review, we will focus on the photo-mediated release and activation of anticancer drugloaded hydrogels, particularly fibrin glue, for the treatment of GBM.

\section{Chemotherapy and the Blood Brain Barrier}

Although chemotherapy has been highly successful in many cancer forms, its use has been disappointing for the treatment of GBM. This is due in a large part to the blood brain barrier (BBB). The BBB is a vital component in protecting the brain against toxins and infections as it limits the movement of blood-borne compounds into the central nervous system (CNS).

The unique impermeability of the BBB is the result of a number of specialized features of the endothelial cells (EC) forming the brain microvasculature, made up of the capillaries and postcapillary venules, meet the unique requirements of the tissue they vascularize as illustrated in figure 1. Firstly, the physical restriction imposed by tight junctions between EC greatly reduces paracellular permeability. Additionally, the transport system regulation of these brain EC limits the number and types of molecules that undergo transcellular transport. For instance, the BBB only allows molecules that are less than $400 \mathrm{Da}$ in size and are highly lipid soluble to passively diffuse 
across this barrier. On the other hand, these specialized EC contain efflux transporters, which transport a wide variety of lipophilic molecules that could otherwise diffuse across the cell membrane, toward the blood. This characteristic is in contrast to the cell membrane found in most cell types. Lastly, the metabolic activity of these endothelial cells, with powerful enzymes metabolizing many potentially harmful substances, adds to the difficulties faced by molecules trying to penetrate the BBB. Therefore, the ability of most chemotherapeutic agents to transverse the BBB is extreamly limited. Since chemotherapy is often administered intravenously, treatment sessions require patients to increase the doses to ensure success. With the requirement of increasing drug dosage to receive adequate drug efficacy, this process ultimately allows for a small therapeutic window, or a small range of clinical benefits outweighing the risks. Eradication of infiltrating glioma cells, though, is a complex challenge since these cells are protected to varying degrees by the blood brain barrier (BBB). In the area of the BAT region, where the invading tumor cells infiltrate into normal brain tissue, the vasculature has been shown to remain intact $[5,6]$. This is critically important since GBM areas in the brain, particularly the tumor infiltration zone, form a significant challenge towards effective treatment $[7,8]$. Since the BBB significantly limits the concentration of chemotherapeutic drugs into the BAT, relatively high systemic drug doses are required. The dose-limiting toxicity of systemically administered agents often leads to inadequate efficacy and unacceptable side effects. In particular, growing attention is now being paid to the potential acute and delayed adverse effects of chemotherapy on brain and cognitive function in patients with both CNS and non-CNS tumors. This debilitating cognitive dysfunction is often referred to as "chemobrain." In contrast to other side effects of chemotherapy, it can persist long after the cessation of treatment.
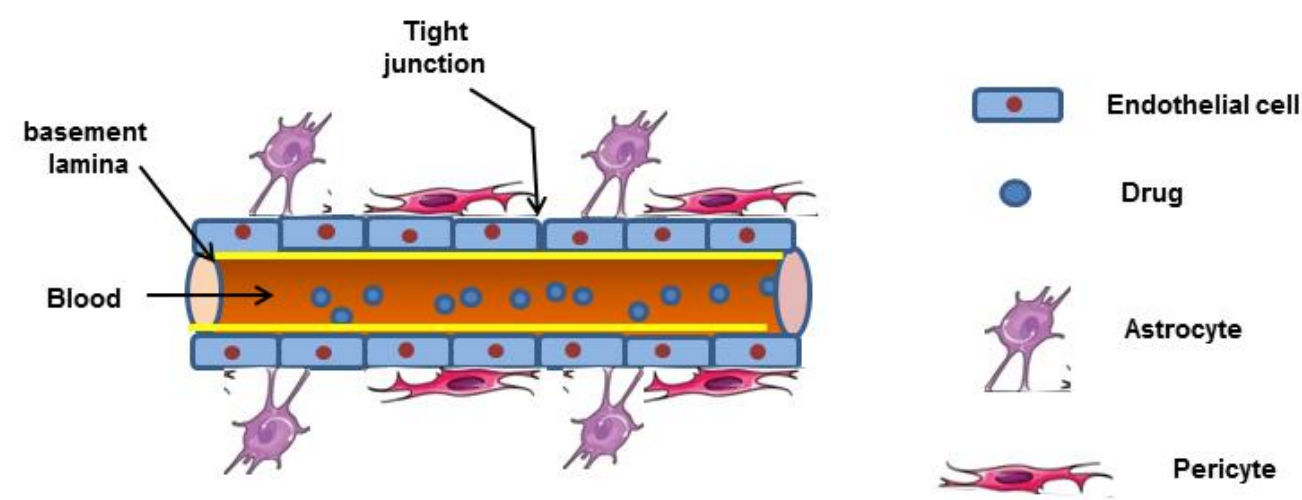

Figure 1 Blood brain barrier simplified overall structure; endothelial cells are connected by tight junctions and share a basement lamina interface with pericytes and astrocytic end-feet.

\section{Localized Drug Delivery Systems (DDS)}

A promising approach, that completely bypasses the BBB, is the local application of chemotherapeutic drugs implanted directly into the resection cavity formed by surgical tumor resection. For this type of local chemptheraputic drug delivery to be most effective they should be delivered in a sustained-release form. DDS implants could take the form of drug-loaded gels, nanoparticles, polymeric films, rods, and wafers. Localized DDS permit increasing the drug dose 
able to reach the remaining infiltrative tumor cells in the brain parenchyma while avoiding systemic side effects. Several types of slow-release vehicles have been evaluated for GBM treatment such as the clinically-approved BCNU biodegradable wafers (Gliadel ${ }^{\circledR}$ ), implanted in the brain cavity after resection surgery. Poor drug penetration in brain tissue, local side effects, drug resistance, implant dislodgement, and infection have demonstrated, in clinical trials, that Gliadel ${ }^{\circledR}$ is sub-optimal for effective GBM therapy [9-11].

The need for other types of sustained-release delivery vehicles that allow triggered drug release and activation is clearly warranted.

\section{Drug Delivery From Hydrogels}

An alternative drug delivery technology under development is the local application of chemotherapeutic agents in the form of various sustained release hydrogels. Comprehensive reviews of the use of hydrogels for the treatment of brain tumors were published by Bastiancich et al. in 2016 and Basso et al. in 2018 [12, 13].

Hydrogels are hydrophilic, polymeric networks able to incorporate large amounts of water. They are stable and swell in aqueous medium such as bodily fluids. In this type of treatment protocol, loaded hydrogels are implanted directly into the resection cavity formed during the surgical procedure of tumor resection $[14,15]$. Drugs can be either directly loaded in the hydrogel matrix or in anticancer nanoagents, which both act as reservoirs to prolong sustained drug release.

Due to their hydrophilic nature, hydrogels have generally been directly loaded with hydrophilic drugs. In contrast, many chemotherapeutic agents are poorly soluble in aqueous media, which results in low incorporation of these drugs in the hydrophilic hydrogels. [16, 17]. To overcome this limitation, hydrophobic drugs can be first in-capsuled or attached to various types of nanoparticles which in turn are incorporated into hydrogel matrixes.

Several types of hydrogels have been studied for the local delivery and slow release of chemotherapeutic drugs in the tumor resection cavity for brain tumors in experimental animals. Bastiancich and co-workers developed a hydrogel made of lipid nanocapsules loaded with lauroylgemcitabine [18]. They demonstrated that in a postoperative rat model, tumor recurrence was significantly delayed by the intracavity deposit of this hydrogel DDS. Extending these results, Zhao et al. developed a sustained-release, photopolymerizable hydrogel containing the anti-cancer drug paclitaxel (PTX) encapsulated in poly (lactic-co-glycolic acid) (PLGA) nanoparticles [19]. Administration of PTX PLGA-NPs-loaded hydrogel into the resection cavity of a GBM orthotropic model led to more than $50 \%$ long-term survival mice (150 days) compared to the control groups (mean survival time 52 days). In a follow-up study, Zhao et al. combined the drug PTX with temozolomide (TMZ) in PLAG nanoparticles incorporated in the photo-polymerizable hydrogel used in their previous report [20]. The reported PTX-TMZ combination suppressed tumor growth more efficiently than the single drugs in the GBM orthotropic tumor model used.

Lin et al. has described the development of MRI-traceable, ultra-thermosensitive hydrogels with rapid gelation ability at $28^{\circ} \mathrm{C}$ for hydrophilic epirubicin (EPI) and hydrophobic paclitaxel (PTX) drugs co-delivered with BSA nanoparticles incorporation [21]. Treatment consisted of surgery plus BSA/PTX-NPs-incorporated hydrogel (Gd/EPI) injected into the resection cavity.

Their results demonstrated the average post-operative survival of ectopic gliosarcoma-bearing 
mice were $100 \%$ at 69 days compared with $0 \%$ at 27 days for controls. Importantly, no tumor recurrences were observed.

\section{Light-Activated Drug Release From Hydrogels}

Although these types of locally-installed, loaded hydrogels provide site specificity, they lack temporal control. Their release kinetics consists of a burst release during the first 24 hours followed by a slower release for a sustained period. The release profile of the drug cannot be controlled once the hydrogel is implanted.

To overcome this limitation, various types of remotely photo-triggered drug delivery systems, that employ light as an external stimulus, have been developed. Four methods of light-activated drug release and/or activation from hydrogels is shown in Figure 2.

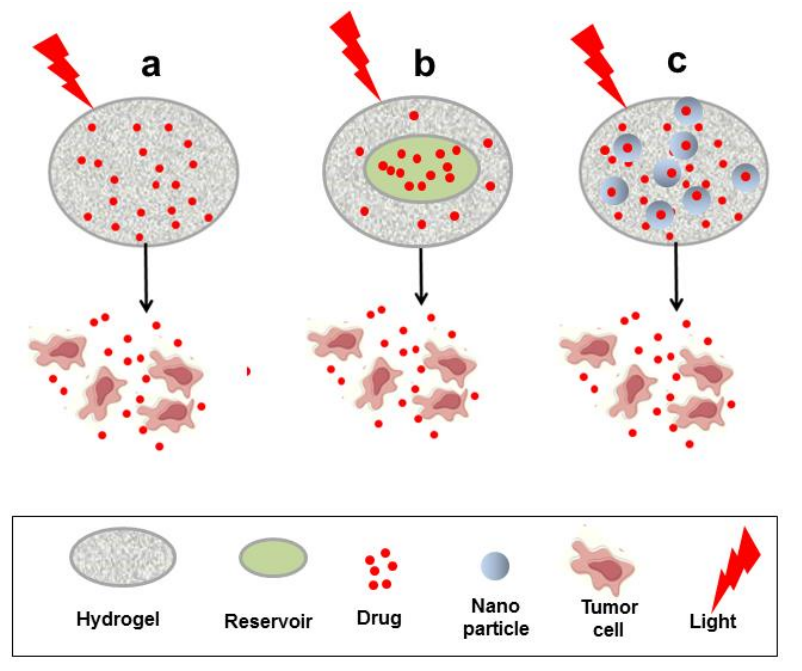

Figure 2 Various forms of light release/activation from hydrogels (a). Drug uniformlydispersed throughout the hydrogel; Released from the hydrogel matrix by light activation. (b) Drug concentrated in central core reservoir surrounded by hydrogel; released from the core by light activation. (c) Drug bound to light sensitive nanoparticles surrounded by hydro gel matrix; released from the nanoparticles by light activation. (d) Uniformly dispersed drug throughout the hydrogel diffuses out, taken up by tumor cells; drug activated by light mediated photochemical internalization.

The use of externally-applied laser light has several advantages such as the ability of being distributed in thin optical fibers and easily-controlled for both wavelength and intensity. One group of light-activated delivery systems relays on the thermal heating effects of near infrared (NIF) irradiation as shown in Figure 2a. The use of NIR irradiation has the advantage of greater depth penetration in tissue compared to visible and UV wavelengths. These systems are often based on thermo-responsive polymers, membranes or hydrogels and some consist of drugs and gold nanoshells/nanorods as NIR to heat converters. Effective release of the embedded drug is obtained upon local heating of the gold nanoparticles via NIR irradiation. Hirbar and co-workers have described a NIR light-responsive, polymer-nanorod composite capable of triggered release of the chemotherapeutic drug doxorubicin [22]. A triggered and stepwise release of drug was seen with multiple cycles of exposure to NIR. Timco et al. developed an implantable drug reservoir 
capped by a nanocomposite membrane whose permeability was temperature-dependent. Irradiation of the device with a NIR laser could produce sustained, on-state drug release for at least 3 hours and could reproducibly deliver short pulses over at least 10 cycles, with an on/off ratio of $30[23,24]$.

Several non-thermal, light-activated DDS encapsulate the drug within a photo-degradable hydrogel, relaying on the photo-induced degradation of the hydrogel matrix to release the cargo $[25,26]$. An important limitation of these relatively simple systems is unwanted drug leakage from the polymer reservoir in the absence of photo-irradiation. Additionally, these types of photodegraded hydrogels allow for only single burst releases, whereas fractionated drug release would be desirable. To overcome non-specific release of drugs in the absence of irradiation, Shah et. al. [27] has reported a photo-activated hydrogel-nanoparticle hybrid scaffold that provides ondemand release of drugs as illustrated in Figure $2 \mathrm{~b}$. Their system consisted of a UV photoactivated compound bound to a chemotherapeutic drug, which is in turn bound to a silica nanoparticle. The drug-conjugated nanoparticles are imbedded in a polymeric hydrogel. Upon exposure to UV light, the activation of the photo-triggered chemical compound initiates a series of intramolecular chemical rearrangements, which cleave the covalently-bound drug from the nanoparticle surface and ultimately releases it from the hydrogel by diffusion. Using an alternating ON/OFF exposure to UV light (30 seconds on, 48 hours off) repeated for four cycles, the drug camptothecin (CPT) release demonstrated a stepwise drug profile. A steep increase in CPT release was observed in the UV-irradiated ON interval with minimal release in the OFF period.

Although this approach shows considerable promise as a remotely-triggerable, reservoir-based drug delivery system, UV irradiation, with its limited depth penetration in tissue $(<1 \mathrm{~mm})$, could prove a barrier to clinical translation. The addition of up-converting (NIR-UV) nanoparticles to this type of hybrid scaffold might help to alleviate this problem [28].

\section{Photo-Activation of Drugs Released From Fibrin Glue}

One form of hydrogel, fibrin glue (FG) composed of a combination of fibrinogen and thrombin, has several characteristics that make it well suited for this form of drug delivery [29]. Unlike most synthetic hydrogels, FG is a natural product and is not just a passive drug delivery matrix. FG has been used clinically in surgery for decades, so its biocompatibility and lack of toxicity well documented [30, 31]. FG consists of two components, a fibrinogen and a thrombin solution rich in calcium. When mixed, the thrombin enzymatically cleaves fibrinogen to form fibrin and factor XIII to factor XIIla, which then crosslinks fibrin to form a gel. Fibrin glue utilized as a drug delivery gel requires addition of exogenous drugs to one of the components prior to their combination.

Fibrin glue has several characteristics that make it an attractive alternative to other forms of hydrogels. Firstly, thrombin and fibrinogen are naturally-occurring substances in the coagulation process and are biodegradable and of low toxicity. FG has been used in surgical procedures for decades and is widely clinically approved. In addition, the parameters of the components can be modified to change the gel's structure, mechanical properties, and degradation [31]. FG is also easily molded to coat the walls of the irregularly-shaped resection cavity, a distinct advantage.

To allow the distribution throughout the solution of fibrinogen and trombin before gel formation, exogenous drugs are added to just one of the components prior to mixture together of the two components. 
Several workers have examined methods to modify fibrin glue's temporal drug delivery kinetics. In studies looking at fibrin glue as a drug delivery vehicle, anti-fibrinolytic agents have been found to slow down FG dissolution. Yoshida et al. conducted a study comparing the release of 5-fluorouracil, mitomycin $\mathrm{C}$ and enocitabine from fibrin glue with and without aprotinin, a compound that inhibits fibrinolysis [32]. In this study, fluorouracil and mitomycin C were released rapidly independent of aprotinin, while enocitabine was released slowly in the presence of aprotinin. They determined that only the hydrophobicity showed a correlation of increasing retention with increasing hydrophobicity. Slower drug release was also achieved in fibrin glue containing sodium alginate. Kitazawa et al. examined the release kinetics of DOX from FG compared to a composite gel of fibrin and sodium alginate [33]. The in vitro mean release time of DOX from FG was 8.7 hours compared to 81 hours for FG containing sodium alginate. This significantly increased sustained release time gives this combined gel a clear therapeutic advantage. Since DOX, the anticancer agent used in these reported experiments, is a cation the negatively-charged sodium alginate is suspected to electrostatically bind to it, causing a more sustained delivery. BLM is also a cation and although not included in this study, is expected to behave similarly. The authors concluded that FG did not directly control the release kinetics. This opens the possibility of the use of FG as a carrier for reservoir or nanoparticle type (Figure $2 b$ and Figure 2c) drug delivery systems.

Anai et al. investigated FG as a drug delivery system for the local administration of Temozolomide (TMZ) in mice bearing subcutaneous tumors induced by the injection of malignant glioma cell lines [34]. TMZ-loaded FG was surgically placed in contact with the tumors. In mice treated with a combination of per os TMZ plus gelled FG-TMZ, the tumors tended to be smaller than in mice treated with TMZ-FG or peros.

TMZ alone. Furthermore, gelled FG-TMZ placed directly on the brain of living mice caused no significant tissue damage either in the acute or chronic phase. Presently, no photo-activated drug release from FG has been reported. FG loaded with the type of light-activated drug-nanoparticles described in reference 27 and shown in Figure $2 c$ might be worthwhile in pursuing.

\subsection{Released Drug Photo-Activation; Photochemical Internalization (PCI)}

Once the drug is released from the locally-implanted hydrogel it needs to enter into tumor cells through their plasma membrane. This limits chemotherapeutic agents to mostly lipophilic or low molecular weight compounds that passively diffuse into the cell cytoplasm. In contrast, many highly-effective chemotherapeutic agents are large and water soluble and are therefore actively transported into cells by endocytosis. Their entrapment in the resulting intracellular endosome/lysosome complex leads to their inactivation. In contrast, if combined with modalities inducing increased endosomal/lysosomal escape, the therapeutic effect of these agents would be significantly increased.

The light-driven drug activation technology, photochemical internalization ( $\mathrm{PCl}$ ) has been shown to enhance the intracellular efficacy of a large variety of chemotherapeutic drugs [35, 36]. In particular, $\mathrm{PCl}$ has been demonstrated to be effective as an adjunct to inadequate surgery and could significantly delay tumor re-growth in a mouse tumor model, compared to PDT or BLM treatment alone [37]. This is an important consideration for postoperative sterilization of the GBM resection cavity. 
Photochemical internalization $(\mathrm{PCl})$ is a technique which utilizes the photochemical properties of PDT for the enhanced and site-specific delivery of drugs into the cell cytoplasm. Figure $3 a$ and $b$ illustrates the $\mathrm{PCl}$ principle.

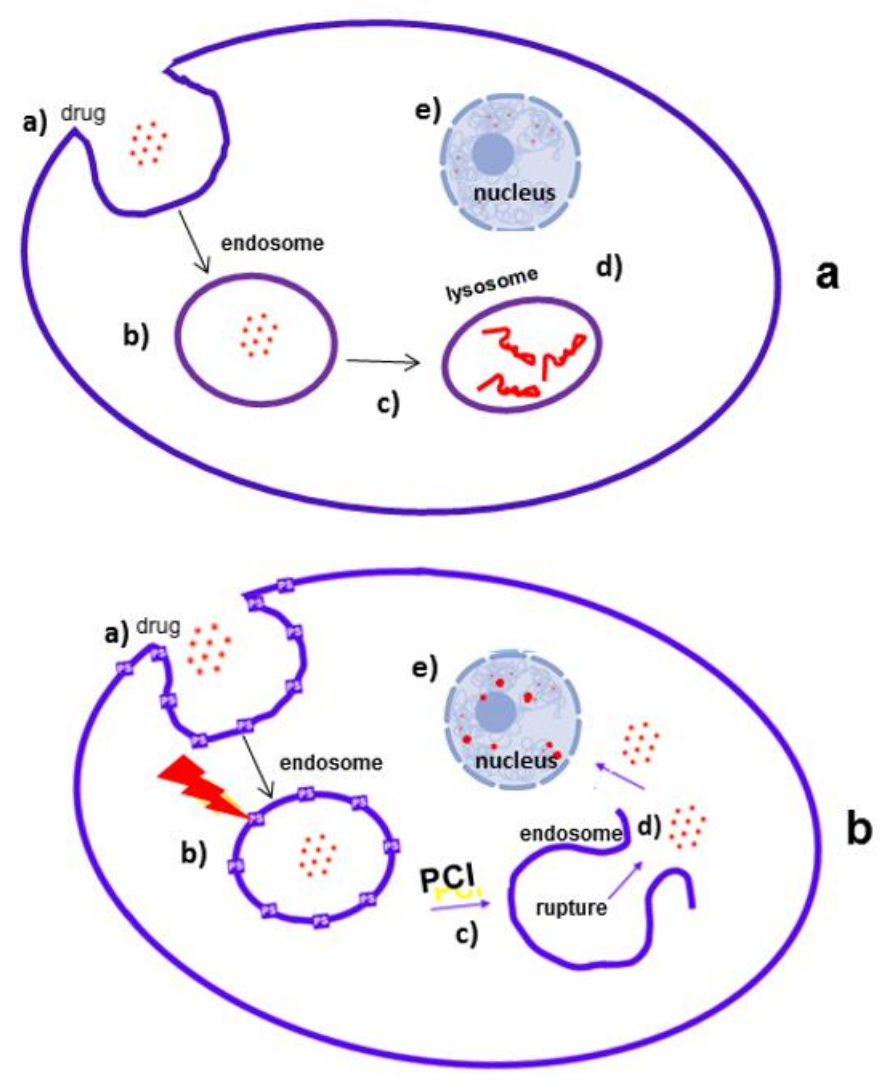

Figure 3 Endosomal escape of drug by photochemical internalization ( $\mathrm{PCl}$ ). (a) a) Drugs that are taken up by endocytosis, b) accumulate in endosome c) endosomal- Iysosome fusion, drug will be degraded by enzymatic hydrolyses d) lysosomal entrapment, e) drug never reaches its target, greatly reducing the therapeutic effect. (b) a) An amphiphilic photosensitizer is given before administration of the drug. b) The photosensitizer binds to the plasma membrane and is taken into the cell together with the drug by endocytosis. The photosensitizer and the drug co-localize in the endosomes. Since the photosensitizer is partly hydrophilic and partly hydrophobic it will remain in the membrane of the endosome, while the drug will localize in the lumen. c) Light exposure leads to photo-induced rupture of the endosome d) subsequent release of the sequestered drug into the cell cytosol e) drug interacts with its target in the nucleus.

$\mathrm{PCl}$ utilizes specific amphiphilic cell membrane-localizing photosensitizers that are transported into the cell via adsorptive endocytosis. The photosensitizer remains in the endosome membrane while the macromolecule is confined to the lumen. The vast majority of $\mathrm{PCl}$ studies have employed several amphiphilic photosensitizers such as aluminium phthalocyanine disulfonate (AIPcS $2 a)$, meso-tetraphenyl porphyrin disulphonate $\left(\right.$ TPPS $_{2 a}$ ) and disulfonated tetraphenyl chlorin (TPCS 2 ; fimaporfin). TPCS $2 \mathrm{a}$ is the only photosensitizer of the three mentioned above that has been used in a PCl clinical study [38]. 
All of these amphiphilic photosensitizers localize in the membranes of endosomes and lysosomes and upon light activation, cause damage to the vesicular membrane. The escaped therapeutic macromolecule will therefore avoid being degraded by lysosome enzymes and can interact with its intended target in the nucleus. Drug activation by $\mathrm{PCl}$ has several clear advantages: 1) There are no restrictions on the size of the molecules that can be effectively delivered, making $\mathrm{PCl}$ highly suitable for a wide variety of drug conjugates, 2) PCI limits the toxic effect of drugs to the sufficiently illuminated regions, sparing normal tissue, 3) PCl is a method that increases the therapeutic efficacy of a wide range of macromolecules allowing for the possibility of using lower drug doses to minimize morbidity, and 4) $\mathrm{PCl}$ can be combined with other therapeutic modalities in a synergistic manner. Therefore, the combination of local, intracavity hydrogels, sustained-release drug delivery, and $\mathrm{PCl}$, as illustrated in Figure $2 \mathrm{~d}$, has the potential of bypassing the BBB, increasing drug concentration in the resection cavity walls, and promoting increased chemotherapeutic efficacy.

Two potent, hydrophilic anti-cancer drugs, bleomycin (BLM) and doxorubicin (DOX), have had limited use for the treatment of GBM. Due to their hydrophilic nature and relatively large size, BLM and DOX have very limited ability to penetrate through the BBB and plasma cell membrane $[39,40]$. Although this is a clear disadvantage in terms of treatment efficacy, these drugs would not re-enter the systemic blood stream, thereby limiting their toxic effects on normal areas of the brain. On the other hand, drugs like BCNU or TMZ, due to their lipophilic character, can re-enter the systemic circulation. $\mathrm{PCl}$ has been demonstrated to significantly enhance the efficacy of BLM and DOX [41-43]. Thus, PCl-mediated BLM or DOX could be highly effective drugs for treatment of GBM employing local hydrogel delivery strategies.

Using a 3-dimensional tumor spheroid model, Nguyen et al. has examined the ability of PCl to enhance the effectiveness of BLM and DOX, released from FG layers [44]. This in vitro model of tumor recurrence simulates the characteristics of avascular tumor nodules and microscopic cell rests in vivo [45]. Spheroid cultures take advantage of homotypic aggregation, a common characteristic of malignant cells. Their natural tendency towards cell-cell adhesion allows for this self-propelled growth in vitro. As the spheroids grow in diameter, they begin to develop a complex cellular environment, directly applicable to what is found in human tumors as shown in figure 4. 


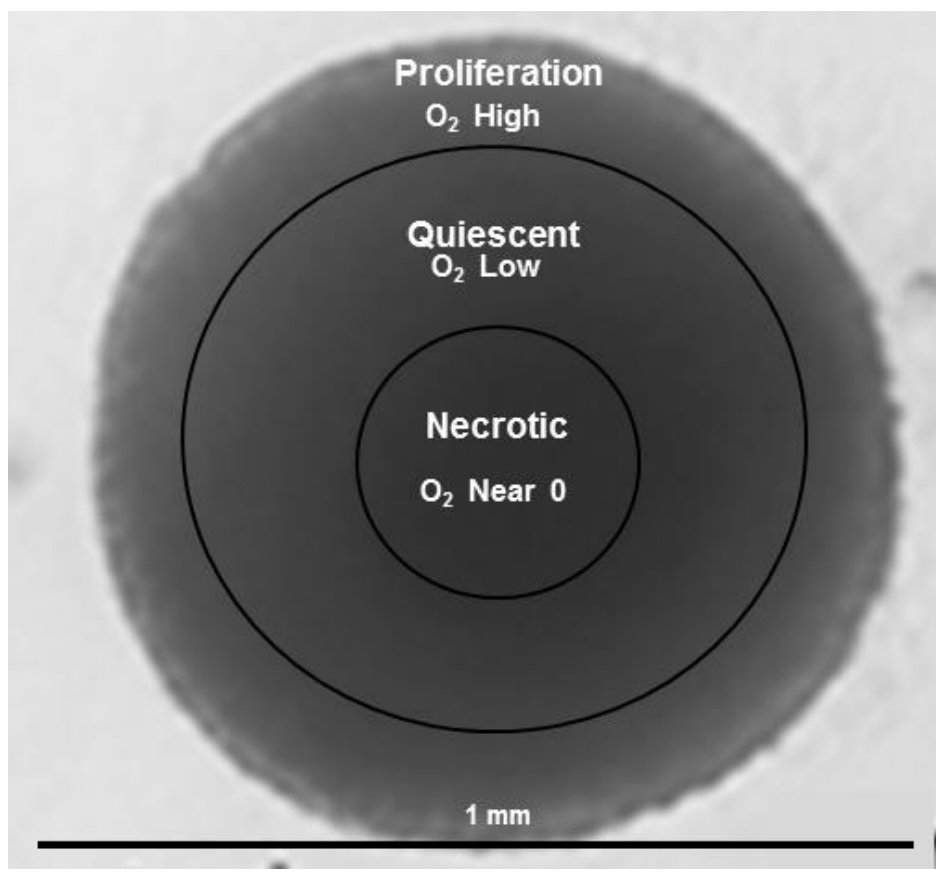

Figure 4 Three zones of cells are commonly found in spheroids as shown in Figure 4. Zone 1, proliferating cells on the periphery and near nutrients and oxygen. These cells are characterized by rapid growth and a high mitotic index, making them susceptible several treatment modalities. Zone 2 quiescent cells, since further away from $\mathrm{O} 2$ supplies are hypoxic, non mitotic and are therefore more resistant to chemo-therapy, ionizing radiation, and $\mathrm{PDT} / \mathrm{PCl}$. Once a spheroid reaches a sufficient size, a necrotic core (zone 3) will develop, comparable to the necrotic center in a typical GBM.

In the reported study, FG layers consisted of a 1:1 ratio of fibrinogen and drug-loaded thrombin. Supernatants covering the FG layers were harvested from the gelled FG containing wells after 2, 24, 48 and 72 hours. Photosensitized glioma 3D spheroids were incubated with BLM or DOX released from the FG layers and exposed to laser light $(\mathrm{PCl}), \lambda=670 \mathrm{~nm}$. Spheroid growth was monitored for an additional 14 days. At the BLM or DOX concentrations used in these experiments to load the FG, spheroid growth was not significantly influenced by the BLM or DOX that was released from the FG gels. In contrast, spheroid growth was significantly inhibited or completely suppressed by $\mathrm{PCl}$ of released drug from either FG-BLM or FG-DOX. Figure 5a shows the effects FG-BLM and FG-BLM PCl on typical spheroid growth inhibition. 
a

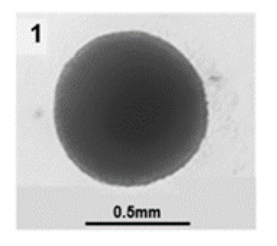

2

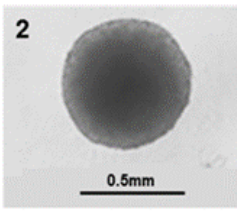

3

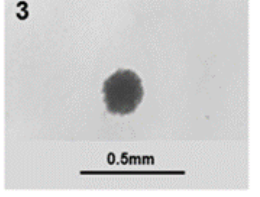

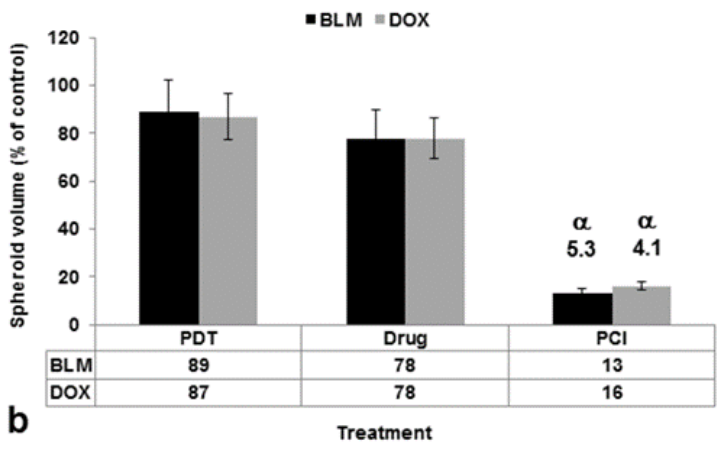

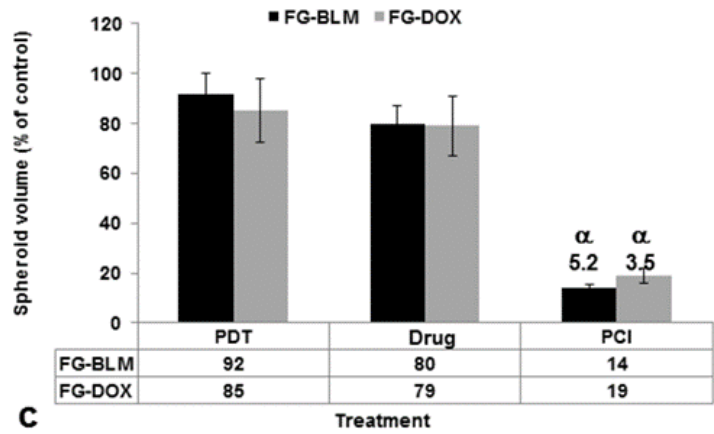

Figure 5 Effects on spheroid growth of BLM/DOX-PCI as free or FG-released drug. a) Light micrograph image of glioma spheroid on day 14 of culture. 1) no treatment control, 2) FG-BLM, FG-BLM-PCl; drug containing supernatants harvested after 24 hours. b) $\mathrm{PCl}$ effects of BLM/DOX as free drug c) PCl effects of BLM/DOX as released drug. Drug-containing supernatant harvested after 72 hours of continuous incubation. Synergistic $\mathrm{PCl}$ effects ( $\alpha$ value) were calculated using normalized values from Figure 5 by multiplying PDT effect $x$ drug effect divided by $\mathrm{PCl}$ effect. If the $\mathrm{PCl}$ effect was synergistic $(\alpha>1)$, antagonistic $(\alpha<1)$, or additive $(\alpha=1)$. $\alpha$ values; shown over $\mathrm{PCI}$ columns, obtained with (b) free or (c) FG released drug.

An important question on the use of $F G$ as a drug delivery vehicle is if the FG-released drug has been degraded compared to free drug. One method to determine drug degradation is to compare the $\mathrm{PCl}$ mediated efficacy and synergy obtained with free or released drug for a given drug concentration. No significant differences were noted in the $\mathrm{PCl}$-mediated efficacy of free drug compared to FG-released BLM or DOX as seen in Figure 5.

The values are shown above the relevant $\mathrm{PCl}$ columns for BLM and DOX. In all cases of free vs FG-released drug, $\alpha$ was greater than one, clearly indicating a synergistic response. $\mathrm{PCl}$ requires an adequate concentration of both drug and photosensitizers in the cancer cells for its therapeutic effects. The constraints on drugs to pass through the BBB also apply to most photosensitizers. Nguyen et al. has examined the ability of the photosensitizer AlPcS2a, released from FG, to induce effective PDT and BLM-PCl [46]. The glioma spheroid model, similar to the one reported for the drug delivery study, was employed for this evaluation. A sequential supernatant harvesting method, with medium replacement following each harvest, was considered as an in vitro simulation for the release that would be promoted in vivo. Their results demonstrate that even after 72 hours and 3 medium changes, there was still sufficient photosensitizer concentration remaining in the supernatant for significant $\mathrm{BLM}-\mathrm{PCl}$ growth inhibition. 


\subsection{Clinical Translation}

The combination of intra-cavity hydrogel sustained-release drug delivery with light activation has the potential of spatial-temporal specific treatment in a limited targeted confined area. Increasing the drug dose allows it to reach the remaining infiltrative tumor cells in the brain parenchyma while avoiding systemic side effects. Localized drug delivery also opens to the use of a variety of complex anti-cancer drugs as well as targeted toxins, consisting of a tumor cell binding antibody/ligand moiety and a protein toxin moiety, that kills the cell. Since these types of macromolecules are too large to cross the BBB they are in turn, when applied locally, prevented from entering the systemic blood stream, thereby limiting their toxic effects on normal areas of the brain. Additionally, local application protects these agents from degradation and clearance until released.

This approach can be readily translated to in vivo animal experiments and eventually to patient protocols, as outlined in Figure 6. To allow for the wound healing process following craniotomy, GBM patients first receive external beam fractioned radiation therapy several weeks after surgical resection. This long treatment delay gives the residual infiltrating tumor cells time to proliferate and form new vascularization, leading to tumor recurrence. In contrast, the instillation of loaded hydrogels combined with indwelling treatment applicators implanted in the resection cavity during the surgical procedure, would allow post-operative treatment to be initiated during this initial critical time period.

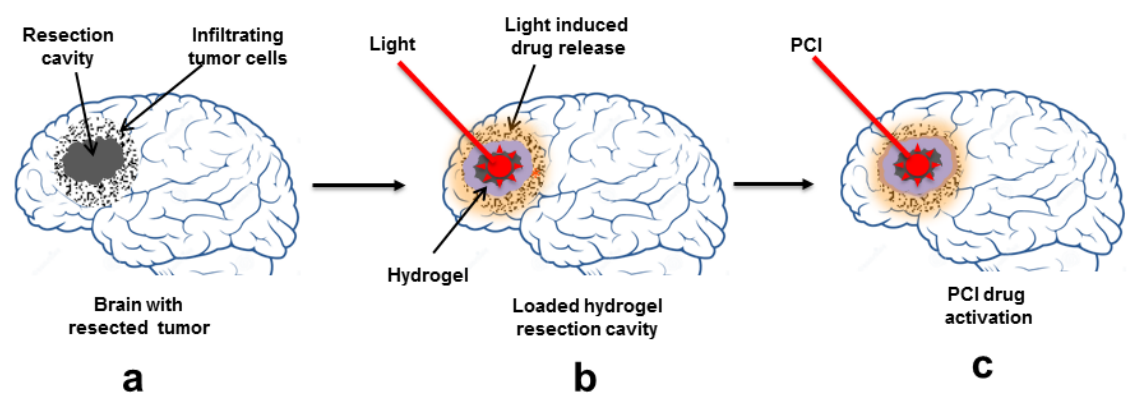

Figure 6 Light induced drug release and activation. a) Following tumor resection infiltrating glioma cells remain in the BAT. b) The resection cavity is coated with drug or drug-photosensitizer loaded hydrogel. Light application releases the drug (orange cloud). c) The efficacy of the released drug will be enhanced by $\mathrm{PCl}$. Photo-activation for both $b$ and $c$ is done through a light applicator, implanted and positioned in the center of the hydrogel-lined resection cavity. (modified and adapted from Nguyen et. al, Insights Neuro Oncol. 2020)

\subsection{Light Delivery}

The basic problem of adequate light treatment in tissue at the various wavelengths required for all of the photo-activated methods shown in Figure 2 can be mediated through an indwelling balloon light or radiation applicator as illustrated in Figure 7. These devices can be implanted in the cavity formed by tumor resection and left in place for an extended period of days [48]. 

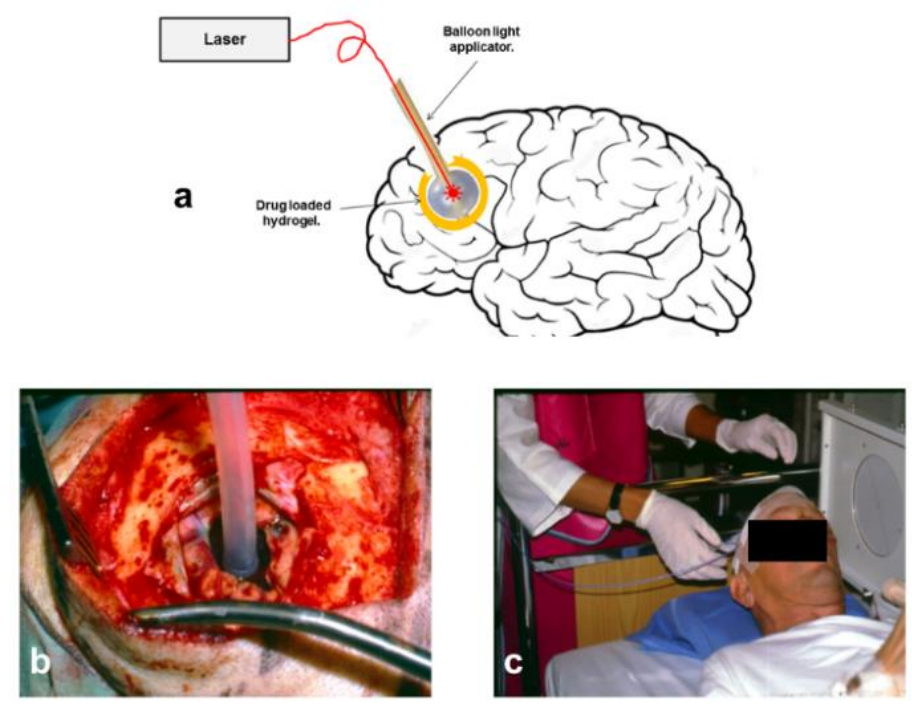

Figure 7 Implantation of an indwelling balloon catheter for use as a radiation or light applicator. a) Illustration of the balloon applicator in a loaded hydrogel coated resection cavity. b) Surgical applicator implantation. The balloon is inflated with saline to fill the cavity and after closure of the operative field the catheter is brought out through the skin and remains in place for several days. c) A patient receiving afterloading brachytherapy (ref. 49) Identical applicators can be used for light delivery for PDT (ref 50), drug release and/or drug activation with PCI. (modified and adapted from Jerjes W et al. J Clin Med. 2020)

These types of indwelling balloon light and after-loading radiation applicators (Figure 7) have been temporarily implanted in patients during surgery, remaining in place in the resection cavity for up to one week, before deflation and removal $[49,50]$. This approach would allow combined light mediated drug delivery and brachytherapy to be commenced shortly after tumor resection in a repetitive/fractionated protocol. Fractionated therapy is often more effective than single shot. In our clinical study of brachytherapy 2 fractions were given each day for 5 days [49]. That should fit well with light administration and a triggered sustained drug release.

\section{Future Perspective}

Although preliminary experimental studies have shown that various drug loaded hydrogels, including FG, are promising adjuvant for the treatment of malignant gliomas, a number of important challenges remain before clinical protocols are initiated. One is unequal and limited targeted drug diffusion into the BAT. One modality under exploration around this problem is the use of nano-medicine-based hydrogels for treating GBM [51]. The various types of light-activated drug release shown in Figure $2 \mathrm{~b}$ and $\mathrm{c}$ could be combined with drug activation via $\mathrm{PCl}$ as illustrated in Figure 6. Another intriguing possibility would be the incorporation, into hydrogels, of factors promoting macrophage migration to the tumor site. Ma can be obtained from the patient, loaded with anti-cancer agents ex vivo, and re-injected back into the Ma donor. Ma are known to actively target and infiltrate brain tumors and can be used as delivery vectors for a variety of anti-cancer agents [44, 52]. Interestingly, preclinical experiments demonstrated that surgery promoted Ma migration into gliomas [53]. 
An important challenge confronting light-activated hydrogel for both drug release and drug activation by $\mathrm{PCl}$ is the limited depth penetration in brain tissue of light. The use of long-duration, low-irradiation light application, as shown for both PDT and $\mathrm{PCl}$, could to a certain extent reduce this limitation by allowing photosensitizer activation at extremely low radiance levels [54]. The possibility of using focused ultrasound (FUS) energy, in place of light, to activate sonosensitizers for sonochemical internalization ( $\mathrm{SCl}$ ) would, for the most part, overcome this problem [55]. FUS has a much greater depth penetration compared to light. Furthermore, since FUS can be applied externally through the skull, in a non-invasive manner, it lends itself to effective, repetitive treatment protocols. Clearly, there is considerable preclinical animal model research remaining to be explored before these strategies can be translated to efficient clinical treatment protocols for patients harboring GBM.

\section{Acknowledgments}

The authors are grateful for the support from the Norwegian Radium Hospital Research Foundation.

\section{Author Contributions}

Background (LN/HH); Chemotherapy and the Blood Brain Barrier (LN/KN/HH); Localized Drug Delivery Systems (DDS)/ Drug Delivery From Hydrogels (AD/EN); Light-Activated Drug Release From Hydrogels (LN/CT); Photo-Activation of Drugs Released From Fibrin Glue (LN/GB/HH); Overall reviewing and corrections $(\mathrm{HH})$.

\section{Funding}

The Norwegian Radium Hospital Research Foundation. Grant nr. SE. 1305/1503.

\section{Competing Interests}

The authors have declared that no competing interests exist.

\section{References}

1. Stummer W, Pichlmeier U, Meinel T, Wiestler OD, Zanella F, Reulen HJ, et al. Fluorescenceguided surgery with 5-aminolevulinic acid for resection of malignant glioma: A randomised controlled multicentre phase III trial. Lancet Oncol. 2006; 7: 392-401.

2. Hirschberg H, Samset E, Hol PK, Tillung T, Lote K. Impact of intraoperative MRI on the surgical results for high-grade gliomas. Minim Invasive Neurosurg. 2005; 48: 77-84.

3. Chamberlain MC. Radiographic patterns of relapse in glioblastoma. J Neurooncol. 2011; 101: 319-323.

4. Dobelbower MC, Burnett III OL, Nordal RA, Nabors LB, Markert JM, Hyatt MD, et al. Patterns of failure for glioblastoma multiforme following concurrent radiation and temozolomide. J Med Imaging Radiat Oncol. 2011; 55: 77-81.

5. Woodworth GF, Dunn GP, Nance EA, Hanes J, Brem H. Emerging insights into barriers to effective brain tumor therapeutics. Front Oncol. 2014; 4: 126. 
6. Van Tellingen O, Yetkin-Arik B, De Gooijer MC, Wesseling P, Wurdinger T, De Vries HE. Overcoming the blood-brain tumor barrier for effective glioblastoma treatment. Drug Resist Updat. 2015; 19: 1-12.

7. Hendricks BK, Cohen-Gadol AA, Miller JC. Novel delivery methods bypassing the blood-brain and blood-tumor barriers. Neurosurg Focus. 2015; 38: E10.

8. Karim R, Palazzo C, Evrard B, Piel G. Nanocarriers for the treatment of glioblastoma multiforme: Current state-of-the-art. J Control Release. 2016; 227: 23-37.

9. Lawson HC, Sampath P, Bohan E, Park MC, Hussain N, Olivi A, et al. Interstitial chemotherapy for malignant gliomas: The Johns Hopkins experience. J Neurooncol. 2007; 83: 61-70.

10. Perry J, Chambers A, Spithoff K, Laperriere N, Neuro-Oncology Disease Site Group. Gliadel ${ }^{\circledR}$ wafers in the treatment of malignant glioma: A systematic review. Curr Oncol. 2007; 14: 189194.

11. Juratli TA, Schackert G, Krex D. Current status of local therapy in malignant gliomas-a clinical review of three selected approaches. Pharmacol Ther. 2013; 139: 341-358.

12. Bastiancich C, Danhier P, Préat V, Danhier F. Anticancer drug-loaded hydrogels as drug delivery systems for the local treatment of glioblastoma. J Control Release. 2016; 243: 29-42.

13. Basso J, Miranda A, Nunes S, Cova T, Sousa J, Vitorino C, et al. Hydrogel-based drug delivery nanosystems for the treatment of brain tumors. Gels. 2018; 4: 62.

14. Norouzi M, Nazari B, Miller DW. Injectable hydrogel-based drug delivery systems for local cancer therapy. Drug Discov Today. 2016; 21: 1835-1849.

15. Bastiancich C, Bianco J, Vanvarenberg K, Ucakar B, Joudiou N, Gallez B, et al. Injectable nanomedicine hydrogel for local chemotherapy of glioblastoma after surgical resection. J Control Release. 2017; 264: 45-54.

16. McKenzie M, Betts D, Suh A, Bui K, Kim LD, Cho H. Hydrogel-based drug delivery systems for poorly water-soluble drugs. Molecules. 2015; 20: 20397-20408.

17. Larrañeta E, Stewart S, Ervine M, Al-Kasasbeh R, Donnelly RF. Hydrogels for hydrophobic drug delivery. Classification, synthesis and applications. J Funct Biomater. 2018; 9: 13.

18. Bastiancich C, Lemaire L, Bianco J, Franconi F, Danhier F, Préat V, et al. Evaluation of lauroylgemcitabine-loaded hydrogel efficacy in glioblastoma rat models. Nanomedicine. 2018; 13: 1999-2013.

19. Zhao M, Bozzato E, Joudiou N, Ghiassinejad S, Danhier F, Gallez B, et al. Codelivery of paclitaxel and temozolomide through a photopolymerizable hydrogel prevents glioblastoma recurrence after surgical resection. J Control Release. 2019; 309: 72-81.

20. Zhao M, Danhier F, Bastiancich C, Joudiou N, Ganipineni LP, Tsakiris N, et al. Post-resection treatment of glioblastoma with an injectable nanomedicine-loaded photopolymerizable hydrogel induces long-term survival. Int J Pharm. 2018; 548: 522-529.

21. Lin FW, Chen PY, Wei KC, Huang CY, Wang CK, Yang HW. Rapid in situ MRI traceable gel-forming dual-drug delivery for synergistic therapy of brain tumor. Theranostics. 2017; 7: 2524-2536.

22. Hribar KC, Lee MH, Lee D, Burdick JA. Enhanced release of small molecules from near-infrared light responsive polymer-nanorod composites. ACS Nano. 2011; 5: 2948-2956.

23. Timko BP, Kohane DS. Prospects for near-infrared technology in remotely triggered drug delivery. Expert Opin Drug Deliv. 2014; 11: 1681-1685.

24. Timko BP, Arruebo M, Shankarappa SA, McAlvin JB, Okonkwo OS, Mizrahi B, et al. Nearinfrared-actuated devices for remotely controlled drug delivery. Proc Natl Acad Sci U S A. 
2014; 111: 1349-1354.

25. Tomatsu I, Peng K, Kros A. Photoresponsive hydrogels for biomedical applications. Adv Drug Deliv Rev. 2011; 63: 1257-1266.

26. Fourniols T, Randolph LD, Staub A, Vanvarenberg K, Leprince JG, Préat V, et al. Temozolomideloaded photopolymerizable PEG-DMA-based hydrogel for the treatment of glioblastoma. J Control Release. 2015; 210: 95-104.

27. Shah S, Sasmal PK, Lee KB. Photo-triggerable hydrogel-nanoparticle hybrid scaffolds for remotely controlled drug delivery. J Mater Chem B. 2014; 2: 7685-7693.

28. Wang X, Xuan Z, Zhu X, Sun H, Li J, Xie Z. Near-infrared photoresponsive drug delivery nanosystems for cancer photo-chemotherapy. J Nanobiotechnology. 2020; 18: 108.

29. Spicer PP, Mikos AG. Fibrin glue as a drug delivery system. J Control Release. 2010; 148: 49-55.

30. Sierra DH. Fibrin sealant adhesive systems: A review of their chemistry, material properties and clinical applications. J Biomater Appl. 1993; 7: 309-352.

31. Radosevich M, Goubran HA, Burnouf T. Fibrin sealant: Scientific rationale, production methods, properties, and current clinical use. Vox Sang. 1997; 72: 133-143.

32. Yoshida H, Yamaoka Y, Shinoyama M, KAMIYA A. Novel drug delivery system using autologous fibrin glue: Release properties of anti-cancer drugs. Biol Pharm Bull. 2000; 23: 371-374.

33. Kitazawa H, Sato H, Adachi I, MAsUKO Y, HORIKOSHI I. Microdialysis assessment of fibrin glue containing sodium alginate for local delivery of doxorubicin in tumor-bearing rats. Biol Pharm Bull. 1997; 20: 278-281.

34. Anai S, Hide T, Takezaki T, Kuroda JI, Shinojima N, Makino K, et al. Antitumor effect of fibrin glue containing temozolomide against malignant glioma. Cancer Sci. 2014; 105: 583-591.

35. Jerjes W, Theodossiou TA, Hirschberg H, Høgset A, Weyergang A, Selbo PK, et al. Photochemical internalization for intracellular drug delivery. From basic mechanisms to clinical research. J Clin Med. 2020; 9: 528.

36. Šošić L, Selbo PK, Kotkowska ZK, Kündig TM, Høgset A, Johansen P. Photochemical internalization: Light paves way for new cancer chemotherapies and vaccines. Cancers. 2020; 12: 165.

37. Norum OJ, Giercksky KE, Berg K. Photochemical internalization as an adjunct to marginal surgery in a human sarcoma model. Photochem Photobiol Sci. 2009; 8: 758-762.

38. Sultan AA, Jerjes W, Berg $K, H \varnothing$ gset A, Mosse CA, Hamoudi R, et al. Disulfonated tetraphenyl chlorin (TPCS2a)-induced photochemical internalisation of bleomycin in patients with solid malignancies: A phase 1, dose-escalation, first-in-man trial. Lancet Oncol. 2016; 17: 12171229.

39. Salford LG, Persson BR, Brun A, Ceberg CP, Kongstad PC, Mir LM. A new brain tumor therapy combining bleomycin with in vivo electropermeabilization. Biochem Biophys Res Commun. 1993; 194: 938-943.

40. Linnert $M$, Gehl J. Bleomycin treatment of brain tumors: An evaluation. Anticancer Drugs. 2009; 20: 157-164.

41. Berg K, Dietze A, Kaalhus O, Høgset A. Site-specific drug delivery by photochemical internalization enhances the antitumor effect of bleomycin. Clin Cancer Res. 2005; 11: 84768485.

42. Mathews MS, Shih EC, Zamora G, Sun CH, Hirschberg H, Blickenstaff J, et al. Photochemical internalization of bleomycin for glioma treatment. J Biomed Opt. 2012; 17: 058001. 
43. Lai PS, Lou PJ, Peng CL, Pai CL, Yen WN, Huang MY, et al. Doxorubicin delivery by polyamidoamine dendrimer conjugation and photochemical internalization for cancer therapy. J Control Release. 2007; 122: 39-46.

44. Shin D, Christie C, Ju D, Nair RK, Molina S, Berg K, et al. Photochemical internalization enhanced macrophage delivered chemotherapy. Photodiagnosis Photodyn Ther. 2018; 21: 156-162.

45. Nguyen L, Shin D, Le MT, Potmaa EO, Idrisa N, Lea JN, et al. Local drug delivery by fibrin glue for glioma treatment: Enhancing drug efficacy by photochemical internalization (PCI). Insights Neurooncol. 2020; 3: 31-42.

46. Madsen SJ, Sun $\mathrm{CH}$, Tromberg BJ, Cristini V, De Magalhães N, Hirschberg H. Multicell tumor spheroids in photodynamic therapy. Lasers Surg Med. 2006; 38: 555-564.

47. Nguyen L, Potma EO, Le JN, Johnson J, Romena G, Peng Q, et al. Photosensitizer delivery by fibrin glue: Potential for bypassing the blood-brain barrier. Lasers Med Sci. 2020. Doi: 10.1007/s10103-020-03140-w.

48. Madsen SJ, Sun CH, Tromberg BJ, Hirschberg H. Development of a novel indwelling balloon applicator for optimizing light delivery in photodynamic therapy. Lasers Med Sci. 2001; 29: 406-412.

49. Johannesen TB, Watne K, Lote K, Norum J, Hennig R, Tveraa K, et al. Intracavity fractionated balloon brachytherapy in glioblastoma. Acta Neurochir. 1999; 141: 127-133.

50. Eljamel MS, Goodman C, Moseley H. ALA and Photofrin ${ }^{\circledR}$ Fluorescence-guided resection and repetitive PDT in glioblastoma multiforme: A single centre Phase III randomised controlled trial. Lasers Med Sci. 2008; 23: 361-367.

51. Ganipineni LP, Danhier F, Préat V. Drug delivery challenges and future of chemotherapeutic nanomedicine for glioblastoma treatment. J Control Release. 2018; 281: 42-57.

52. Madsen SJ, Hirschberg H. Macrophages as delivery vehicles for anticancer agents. Ther Deliv. 2019; 10: 189-201.

53. Zhu H, Leiss L, Yang N, Rygh CB, Mitra SS, Cheshier SH, et al. Surgical debulking promotes recruitment of macrophages and triggers glioblastoma phagocytosis in combination with CD47 blocking immunotherapy. Oncotarget. 2017; 8: 12145-12157.

54. Shin D, Nguyen L, Le MT, Ju D, Le JN, Berg K, et al. The effects of low irradiance long duration photochemical internalization on glioma spheroids. Photodiagnosis Photodyn Ther. 2019; 26: 442-447.

55. Hirschberg $H$, Madsen SJ. Synergistic efficacy of ultrasound, sonosensitizers and chemotherapy: A review. Ther Deliv. 2017; 8: 331-342.

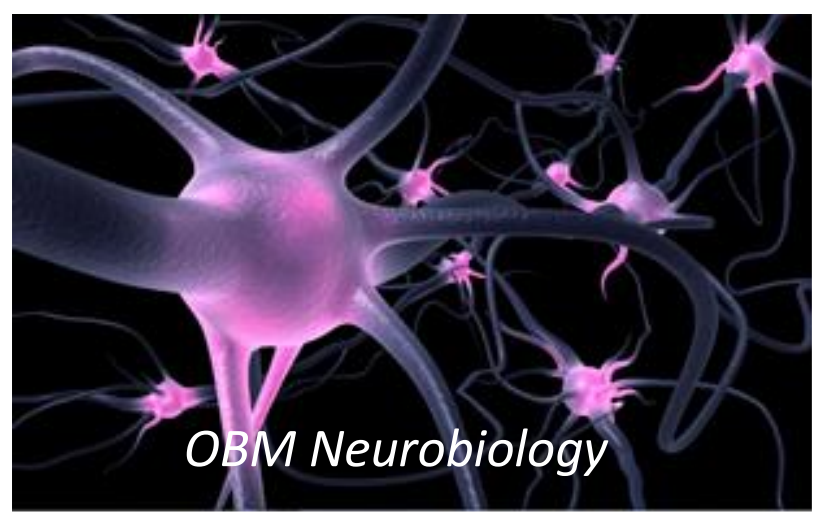

Enjoy OBM Neurobiology by:

1. Submitting a manuscript

2. Joining volunteer reviewer bank

3. Joining Editorial Board

4. Guest editing a special issue

For more details, please visit:

http://www.lidsen.com/journals/neurobiology 